\title{
Habilidades cognitivas y aprendizaje significativo de la adición y sustracción de fracciones comunes
}

\section{Cognitive skills and meaningful learning of addition and subtraction of common fractions}

ISSN 1510-2432 - ISSN 1688-9304 (en línea) - DOI: https://doi.org/10.18861/cied.2016.7.2.2610

Rubicel Manuel Capilla

Doctor en Ciencias de la Educación, Universidad Santander, México. Licenciado en Educación Primaria, Escuela Normal Urbana Federal "J. Jesús Romero Flores", México. Maestro en Educación y Asesor académico en modelos de pensamiento y construcción de conocimiento, y desarrollo de habilidades y estrategias cognitivas, Universidad Pedagógica Nacional, México. Investigador en temas cuantitativos, cualitativos, experimental, práctica docente y procesos de aprendizaje.

Fecha de recibido: 17/03/2016

Fecha de aceptado: 10/10/2016

\section{Resumen}

La investigación tuvo como finalidad identificar la correlación y el impacto que existen entre la equivalencia, comparación y orden de fracciones -considerando a estos como habilidades o procesos cognitivos-y el aprendizaje significativo de la adición y sustracción de fracciones comunes. En este sentido se planteó la interrogante: ¿Cuál es la correlación e impacto de las habilidades cognitivas con respecto al aprendizaje significativo de la adición y sustracción de fracciones comunes? Se adoptó un enfoque cuantitativo y un diseño cuasiexperimental, longitudinal y correlacional con una muestra conformada por 17 estudiantes de tercer grado de telesecundaria. Para medir las variables se aplicaron dos cuestionarios de indagación equivalentes en su contenido con preguntas cerradas o diferenciados en los dígitos con el fin de garantizar la confiabilidad de los resultados aplicados en la fase de la preprueba y postprueba, respectivamente. En la fase intermedia se aplicó una secuencia de aprendizaje con la finalidad de desarrollar las habilidades cognitivas referidas a partir de actividades lúdicas y situaciones problema, así como el uso de recursos concretos y manipulables. Los resultados revelan una fuerte correlación positiva entre las variables habilidad cognitiva y operaciones con fracciones, lo que supone que el desarrollo de las habilidades cognitivas cataliza positivamente el aprendizaje significativo de las operaciones matemáticas referidas y viceversa.

Palabras clave: procesos cognitivos, adición de fracciones, sustracción de fracciones, aprendizaje significativo.

\begin{abstract}
The research had the objective of identifying the correlation and impact existing between equivalence, comparison and order of fractions -considering them as skills or cognitive processes- and meaningful learning of addition and subtraction of common fractions. In this sense, the following question was raised: What is the correlation and impact of cognitive skills in respect of meaningful learning of the addition and subtraction of common fractions? A quantitative approach and quasi-experimental, longitudinal and co-relational design were adopted, with a sampling comprised of 17 students attending lower secondary tele-education. To measure the variables, two survey questionnaires were applied that
\end{abstract}


are equivalent in content, with closed questions or differentiated in digits to ensure reliability of the results applied at the stages of the pretest and posttest, respectively. In the intermediate phase, a sequence of applied learning was applied in order to develop the aforementioned cognitive skills from recreational activities and problem situations as well as the use of concrete and controllable resources. The results reveal a strong positive correlation between the variables of cognitive skill and fraction operations, which presumes that the development of cognitive skills positively catalyze meaningful learning of the said mathematical operations and vice versa.

Keywords: cognitive processes, adding fractions, subtracting fractions, meaningful learning.

\section{Introducción}

El objetivo de los sistemas educativos actuales consiste en dotar al sujeto de herramientas que le permitan construir y reconstruir constantemente saberes diversos por sí mismo. Las habilidades cognitivas representan una de estas herramientas o medios y el sujeto recurre a las mismas para identificar y transformar la información en conocimiento. A tal fin es menester el desarrollo de procesos cognitivos tales como la observación, la atención y el procesamiento, los que involucran el análisis, síntesis, comparación y ordenamiento entre otros, así como el almacenamiento y la recuperación.

Dichas habilidades cognitivas también adquieren relevancia toda vez que dan origen a otras más específicas relacionadas con un área disciplinar o campo de conocimiento determinado o incluso con tareas específicas dentro de las mismas. Asimismo, su desarrollo favorece el aprendizaje significativo de conocimientos que han sido validados por la cultura y que son necesarios para desenvolverse en ella, tal es el caso de la adición y sustracción de fracciones comunes.

Por un lado, se advierte la necesidad de centrar la actividad educativa en la promoción y desarrollo de tales procesos cognitivos en los sujetos que cursan su educación básica. Por otro, debe resaltarse la lógica de considerar a tales procesos como base fundamental para el desarrollo de competencias matemáticas.

\section{Problema}

Es común identificar en alumnos que cursan los últimos grados de educación básica la dificultad para resolver correctamente la adición y sustracción de fracciones comunes, bien por medio del algoritmo o por procesos no convencionales. A partir de un estudio preliminar se halló que el origen de esta dificultad radicaba en la ausencia de sentido y significado de tales dígitos numéricos para el alumno. A efectos de profundizar en esta problemática se diseñó y aplicó un diagnóstico en el que los datos proyectados por el mismo indicaron, por un lado, que el alumno presenta un bajo nivel de desarrollo de habilidades cognitivas propias para el manejo de fracciones comunes y la resolución de operaciones matemáticas, y por otro, los procesos de enseñanza soslayan el desarrollo de tales habilidades cognitivas. Las mismas dejan de concebirse como medios importantes para la consecución de aprendizajes diversos y se inscriben únicamente en la transmisión de saberes conceptuales.

Bajo esta premisa, con la finalidad de adoptar elementos empíricos y teóricos que permitan resaltar el impacto del desarrollo de habilidades de pensamiento en los procesos de adquisición de conocimientos con sentido y significatividad y, en consecuencia, la promoción de competencias matemáticas, la investigación giró en torno a la identificación y la correlación de las variables habilidades cognitivas y aprendizaje significativo de la adición 
y sustracción de fracciones comunes. Para ello fue menester el planteo de las siguientes interrogantes: ¿Cuál es el impacto de las habilidades cognitivas sobre el aprendizaje significativo de la adición y sustracción de fracciones comunes?, ¿Cuáles son las habilidades cognitivas que facilitan la resolución de fracciones comunes?, ¿Cuáles son las habilidades cognitivas que propician el aprendizaje significativo de adición y sustracción de fracciones comunes?, ¿Qué habilidades cognitivas debe desarrollar el alumno para resolver tales operaciones matemáticas?

\section{Antecedentes}

Parra y Flores (2008) analizaron los significados que los alumnos con bajo aprovechamiento escolar tenían de los conceptos relacionados con la fracción y los recursos utilizados para solucionar problemas matemáticos y caracterizaron la interacción suscitada entre ellos. Con respecto al manejo de fracciones comunes, identificaron que las dificultades se inscriben en las concepciones previas y la falta de correspondencia entre el signo y el significado culturalmente compartido. Reconocieron que el trabajo cooperativo y la contextualización favorecen el desarrollo cognitivo, manifestado en la habilidad para argumentar las propuestas de solución de una situación problema.

Perera y Valdemoros (2009) enmendaron esta dificultad con actividades realistas, lúdicas y colaborativas. Lograron con ello el desarrollo intelectual de los niños, habilitándolos para que ellos mismos construyeran sus propios conocimientos sobre la base de sus experiencias cotidianas, expresaran simbólicamente una fracción y establecieran la relación de orden y equivalencia de fracciones, así como también lograron incrementar su habilidad para manipular mentalmente este tipo de dígitos. En tal sentido Terán de Serrantino \& Pachano (2009) probaron la influencia de la enseñanza matemática realista, lúdica y colaborativa, y la resolución de problemas en la conformación de las nociones de fracción en la educación básica bajo el paradigma constructivista. Concluyeron que las actividades bajo estas características tienen un impacto favorable, de manera tal que no solo favorecieron la formación de las nociones referidas sino también lograron aumentar la motivación e interés en los alumnos por el contenido matemático referido.

Por un lado, las investigaciones anteriores retoman la problemática del aprendizaje de las fracciones, que se centra en las nociones que poseen los alumnos así como los requisitos y las condiciones que se requieren para favorecer la enseñanza y aprendizaje de contenido curricular. Por otro lado refuerzan los planteamientos de Ausubel, Novak \& Hanesian (2009) en torno al aprendizaje significativo. Este autor que refiere a que el aprendizaje debe basarse en las experiencias previas del sujeto. Asimismo resalta que en el acto de construir significados propios subyace la importancia de reconstruir o modificar estos de manera continua a fin de establecer la correspondencia de signo y significado, de construir el significado real del objeto y de construir significados colectivos y compartidos entre los sujetos.

\section{Propósitos}

El propósito de la investigación radicó en identificar la correlación -dependiente e independiente, respectivamente- entre las variables habilidades cognitivas y operaciones con fracciones comunes. La primera variable se estructuró con las dimensiones equivalencia de fracciones, comparación de fracciones y orden de fracciones. Para la segunda variable las dimensiones fueron adición de fracciones comunes y sustracción de fracciones. A tal efecto se redactó la hipótesis de trabajo correlacional estadístico afirmando que la 
correlación entre habilidad cognitiva y operaciones con fracciones comunes no es igual a cero. Los resultados indicaron una correlación positiva entre las variables, lo que comprobó la hipótesis.

\section{Fundamentación teórica}

\section{Aprendizaje significativo}

El aprendizaje significativo es una teoría cognitiva del aprendizaje. Responde a dos principales preguntas. La primera consiste en explicar cómo se adquiere el conocimiento y la segunda en cómo se retiene. Concede importancia a los procesos en los que el individuo se auxilia para aprender, así como a las condiciones necesarias para que este tipo de aprendizaje se produzca. Dicha teoría se inserta en el Constructivismo y postula la premisa de que es el individuo mismo el que genera y construye su propio aprendizaje. Pozo (2006) afirma que esta teoría es del tipo cognitivo, organicista y de reestructuración y sitúa su atención en el aprendizaje suscitado en contextos escolares. Destaca la importancia de conocer no solo los procesos mentales que ejecuta el individuo para aprender nuevos saberes sino también cómo mejorarlos.

Según Ausubel, Novak \& Hanesian (2009) el aprendizaje significativo ocurre cuando nuevas ideas, conceptos y proposiciones pueden ser aprendidos de manera significativa en la medida en que otros conceptos, ideas o proposiciones relevantes estén claros y disponibles en la estructura cognitiva del individuo. De esta manera el sujeto establece una relación sustantiva entre los conceptos que posee y la nueva información. Por tal razón, el autor sugiere ubicar los conocimientos que trae consigo el individuo en torno al contenido. Dichos conocimientos tienen relevancia para que se concrete la relación sustantiva con la nueva información. El nuevo objeto de aprehensión debe ser relacionable con la información preexistente en el sujeto, es decir, debe cumplir con el criterio de relacionalidad.

Para el caso que aquí se aborda, las experiencias previas y la estructura cognitiva del alumno se manifiestan no solo en la noción, concepción, significado que posee y en la forma de representar una fracción común sino que también se advierten en la capacidad para observar, recordar, relacionar, ordenar, comparar y establecer equivalencia de fracciones comunes, habilidades cognitivas específicas que determinan el aprendizaje significativo de la adición y sustracción de tales números racionales.

La estructura cognitiva, representada por las ideas o conceptos existentes funciona como anclaje para establecer la conexión significativa con nuevos conceptos. Empero, este proceso no es unidireccional sino bidireccional dado que tanto los conceptos o ideas preexistentes como los nuevos que se integran evolucionan conformando una nueva estructura. Es decir, cuando se concreta la conexión lógica y sustancial entre un concepto prexistente en la estructura cognitiva y una nueva información o concepto presentado, este segundo adquiere significado, lo que favorece que ambos evolucionen. A este proceso Ausubel, Novak \& Hanesian (2009) denominan Principio de asimilación. Así, al retomar el contenido curricular que aquí se aborda, la noción o concepto de fracción presente en la estructura cognitiva del alumno servirá de subsunsor para la aprehensión significativa del concepto de fracción equivalente. Esta, a su vez, permitirá comprender que una fracción puede representarse simbólicamente con diferentes dígitos. En este mismo sentido, la adición de números naturales será el subsunsor para el concepto de adición de fracciones comunes, y este segundo concepto permite comprender que la adición no se limita a los números naturales, se extiende a las fracciones comunes. 
La relación sustantiva que establece el sujeto entre los conceptos previos y la nueva información se establece solo cuando el objeto de aprehensión es significativamente lógico, es decir, es posible establecer la conexión con la estructura cognitiva. En este sentido, los nuevos conceptos a presentar deben ser relacionables con las ideas o conceptos que ya posee el sujeto. Asimismo, cuando la conexión o relación entre los conceptos se concreta se construye un significado psicológico y pasa a formar parte de la nueva estructura cognitiva. El autor destaca otro de los factores que intervienen en la apropiación del significado lógico y psicológico: el interés del sujeto por aprender un nuevo saber. Ausubel, Novak \& Hanesian (2009) destacan que este tipo de aprendizaje se caracteriza por lograrse a partir de los intereses, la motivación y necesidades del individuo. Por ello sugieren el reconocimiento de tales intereses dado que no son los mismos para todos los alumnos en torno a un contenido curricular.

En síntesis, el aprendizaje significativo es la adición de subsumidores o conocimiento previo, estructura cognitiva e interés o disposición. La ausencia de uno de los sumandos impacta en esta ecuación. Así, por ejemplo, la ausencia del interés del alumno o la estructura cognitiva que demanda un conocimiento disciplinar determinan la calidad del aprendizaje significativo que construya en sujeto. Más concretamente, cuando el alumnado no ha desarrollado la estructura cognitiva requerida para comprender una idea, un concepto o para aplicar una habilidad cognitiva, la nueva información carecerá de significado.

Esta teoría resalta tres tipos de aprendizaje: de representaciones, conceptos y proposiciones. En el primero el sujeto atribuye significado a los símbolos. En el segundo, incluye el aprendizaje de "objetos, eventos, situaciones o propiedades de que posee atributos de criterios comunes y que se designan mediante algún símbolo o signo" (Ausubel, Novak \& Hanesian, 2009). Aquí el sujeto aprende conceptos a partir de dos procesos: formación y asimilación. La formación se concreta a partir de la experiencia directa y la segunda a partir de la acumulación de vocabulario. El tercer tipo de aprendizaje se concreta cuando el sujeto logra captar el significado de varias palabras relacionadas entre sí.

Finalmente, en la construcción de conocimientos no solo intervienen factores intrapsicológicos tal como señala Coll (1990), sino que en este tipo de aprendizaje también intermedian factores interpsicológicos, los que se insertan en la cultura. Los individuos que conforman el grupo social y conocimientos generados dentro de este son partes medulares de la cultura. Aquí se construyen y comparten los distintos saberes, los signos y significantes. A éstos debe acceder el sujeto, a fin de que logre desenvolverse satisfactoriamente dentro de la cultura dado que dichos conocimientos, signos y significantes son las herramientas interpsicológicas. Ambos tipos de factores referidos son mediadores entre el conocimiento u objeto y el sujeto.

En este orden de ideas, el grupo escolar favorece tal acción. El grupo de individuos al cual pertenece logrará desenvolverse de la mejor manera, lográndose un mejor desarrollo de la comunidad y sociedad en general. Lo tratado con antelación corresponde a la acción del individuo en el proceso de aprendizaje. Sin embargo, es menester considerar al otro de forma igualmente importante en el proceso de enseñanza-aprendizaje. En la enseñanza el papel del mediador es relevante en tanto que determina también el buen acceso hacia el aprendizaje significativo. 


\section{Procesos cognitivos}

En relación a los procesos cognitivos se abordan someramente tres tipos: generales, particulares y específicos. Los dos primeros se abordan para explicar los procesos mentales que el sujeto aplica para la adquisición de conocimientos diversos. Los específicos se aluden para destacar los procesos mentales que demanda el aprendizaje significativo de la adición y sustracción de fracciones comunes.

De acuerdo con Pozo (2006) y Rivas (2010) la teoría del aprendizaje significativo además de sustentarse en las teorías psicogenética y sociocultural, también recupera premisas centrales de la teoría del procesamiento de la información retomando la premisa de los procesos cognitivos generales: recopilación de información, conexión entre la nueva información y la existente, recuperación y aplicación de la información.

En estos procesos cognitivos generales intervienen procesos mentales específicos tales como observar, recordar, ordenar, analizar y evaluar, entre otros. Dichos procesos determinan cualitativa y cuantitativamente la construcción de significados. ¿Cómo interactúan en el proceso de aprender significativamente y cómo se pueden considerar en el proceso didáctico?

La cuestión planteada puede ser abordada desde la perspectiva del procesamiento de la información a partir de dos momentos principales: la entrada y salida de información, respectivamente (input y output). Empero, en este proceso es posible identificar tres momentos y procesos cognitivos.

En el primer momento de aprendizaje algunos de los procesos cognitivos que intervienen son observar (que implica dar una dirección intencional a nuestra percepción), atender, identificar, buscar y encontrar datos, elementos u objetos, recordar, recuperar la información almacenada en la memoria a largo plazo y relacionar (establecer la conexión de una idea, concepto, hecho o situación con otro).

En el segundo momento de construcción de saberes intervienen procesos cognitivos tales como:

- Ordenar (entendido como la acción de disponer de manera sistemática un conjunto de datos a partir de un atributo determinado). Implica subhabilidades tales como reunir, agrupar, listar y seriar;

- Comparar, que implica establecer la relación de semejanza y diferencia entre objetos, conceptos o hechos que ayudan a relacionar o establecer una mayor conexión entre la información del exterior y la que posee el sujeto aprehensor.

En el tercer momento los procesos cognitivos que favorecen la salida de la información -que comprueban no solo la conexión sino también el almacenaje en la memoria a largo plazo, la recuperación de la información y la adquisición de sentido y significados, es decir, la comprensión del objeto de conocimiento- son los siguientes:

- Analizar. Implica destacar los elementos básicos de una unidad de información y contempla subhabilidades tales como comparar, destacar, distinguir y resaltar;

- Aplicar. El sujeto es capaz de utilizar los conceptos e ideas en situaciones reales y específicas;

- Evaluar. Consiste en valorar a partir de la comparación entre un producto, los objetivos y el proceso. Implica subhabilidades tales como examinar, criticar, estimar y juzgar. 
Los procesos cognitivos específicos que intervienen en el aprendizaje significativo de la adición y sustracción de fracciones comunes son los siguientes:

- Comparar fracciones comunes. Puede observarse cuando el individuo distingue, entre dos o más números de esta índole, la diferencia de magnitud o cantidad que representan cada una de estas fracciones, es decir, consiste en determinar mediante los signos $>$ (mayor que) y < (menor que) si una fracción común es menor o es mayor en relación a otra. Ej.: $\frac{1}{\mathbf{3}} \frac{1}{\mathbf{3}}>\frac{1}{\mathbf{6}} \frac{\mathbf{1}}{\mathbf{6}}$

- Ordenar. Esta habilidad cognitiva consiste en situar en un lugar una serie de fracciones comunes de acuerdo a la cantidad que representen, es decir, acomodarlos de menor a mayor o viceversa. Ej.: $\frac{1}{5,1}, \frac{2}{6}, \frac{2}{4}, \frac{4}{7}, \frac{3}{5} \frac{1}{5,1}, \frac{2}{6}, \frac{2}{4}, \frac{4}{7}, \frac{3}{5}$

- Comparar y ordenar fracciones. Favorece la habilidad cognitiva de equivalencia de fracciones. Implica demostrar que una fracción común representa la misma cantidad en relación a otras. Se indica mediante el uso del signo $=$. Ej.: $\frac{\mathbf{1}}{\mathbf{3}} \frac{\mathbf{1}}{\mathbf{3}}=\frac{\mathbf{2}}{\mathbf{6}} \frac{\mathbf{6}}{\mathbf{6}}$.

En suma, estos procesos en conjunto actúan en la adquisición de sentido numérico de las fracciones comunes, por lo que es menester que el sujeto desarrolle los procesos generales, particulares y específicos, pues a partir de los mismos construirá conceptos, estructuras cognitivas, conocimientos y aprendizajes significativos relativos a la adición y sustracción de fracciones comunes.

\section{Método}

El estudio contempló 17 alumnos cursantes del tercer grado de secundaria de la escuela Telesecundaria "ESTV0295-Y" ubicada en la comunidad indígena de Ocumicho, Municipio de Charapan, Michoacán.

La muestra fue de tipo no probabilístico y criterial. El primer criterio de inclusión consideró a alumnos inscritos en la escuela referida durante el ciclo escolar 2013-2014, integrantes del $3^{\circ}$ grado, grupo A y que hubieran participado en la fase de la preprueba. Un segundo criterio fue la exclusión, aplicado a alumnos que no participaron en la fase de la preprueba. El tercer criterio, eliminación, consistió en no incluir en la muestra a alumnos que hubieran faltado una o más veces al momento de la aplicación de las secuencias de aprendizaje así como el día de la aplicación de la posprueba.

Tanto en la fase de preprueba como en la de posprueba se aplicaron dos instrumentos: un cuestionario y una Escala Likert. El cuestionario consideró un total de 12 reactivos: 6 para recabar datos concernientes a las habilidades cognitivas, 2 para cada habilidad. El puntaje total del instrumento correspondió a 24 puntos: 1 punto para la respuesta incorrecta y 2 puntos para la respuesta correcta. Los reactivos de este apartado contemplaron ejercicios que demandaban al alumno el manejo de fracciones comunes y la colocación de un dígito o fracción según correspondiese, siempre cuidando el cumplimiento de condiciones demandas por los signos =, > y < (igual que, mayor que y menor que), es decir, para establecer la equivalencia, comparar y ordenar fracciones. Los 6 reactivos restantes se distribuyeron equitativamente para la adición y sustracción de fracciones comunes. Los mismos demandaron al alumno la colocación de un digito para completar la fracción 
correcta o en su caso los dígitos que conforman una fracción, la colocación del denominador o numerador o en su caso ambos que cumpliese la condición.

Los reactivos únicamente aceptaban una respuesta correcta. Asimismo incluyeron el manejo de fracciones que frecuentemente se utilizan tanto dentro como fuera del ámbito escolar, así como aquellos a los que, de manera esporádica, se recurre en ambos espacios.

La validez de los cuestionarios se realizó en función del contenido, dado que este midió las variables identificadas en el marco conceptual o teórico (Hernández, Fernández \& Baptista, 2010). La confiabilidad se basó en el método de mitades partidas: por cada habilidad cognitiva y operación matemática involucrada se consideró la inclusión de dos reactivos, es decir que los instrumentos contemplaron dos mitades totalmente equivalentes. Otro de los elementos considerados en la adopción de este método fue la aplicación de cada uno de los cuestionarios en una sola oportunidad.

La Escala Likert se utilizó para recopilar y organizar los datos que proyectaron los cuestionarios aplicados tanto para la preprueba como para la postprueba. También permitió interpretar, graficar e identificar la correlación de variables con el mismo tipo, características y número de reactivos. Únicamente se le modificó al anexarle la forma de registrar las respuestas para indicar si el alumno logra resolver correctamente las operaciones matemáticas por lo que solo es aceptable registrar una respuesta (sí o no). Los reactivos y la forma de registrar el dato que proyecta el alumno se presentan en el cuadro $\mathrm{N}^{\circ} 1$.

Tabla $\mathbf{N}^{\circ}$ 1. Plan de registro de resultados de los reactivos

\begin{tabular}{|l|l|l|l|l|}
\hline \multicolumn{1}{|c|}{ ASPECTO A EVALUAR } & \multirow{2}{*}{$N^{\circ}$ ÍTEM } & \multicolumn{2}{|c|}{ DIMENSIÓN } & \multicolumn{2}{c|}{ RESPUESTA } \\
\cline { 3 - 5 } & & & NO=1 & Sí=2 \\
\hline Habilidad cognitiva & $1-2$ & Equivalencia & & \\
\cline { 2 - 5 } & $3-4$ & Comparación & & \\
\cline { 2 - 5 } & $5-6$ & Orden & & \\
\hline $\begin{array}{l}\text { Operaciones con } \\
\text { fracciones comunes }\end{array}$ & $7-9$ & Adición de fracciones comunes & & \\
\cline { 2 - 5 } & $10-12$ & $\begin{array}{l}\text { Sustracción de fracciones } \\
\text { comunes }\end{array}$ & & \\
\hline
\end{tabular}

Los criterios utilizados para la validación y confiabilidad de este segundo instrumento fueron los de Contenido y Método de Mitades. Se utilizaron el coeficiente de Guttman, Spearman-Brown y Alfa de Cronbach cuyos resultados fueron de 0.898, 0.916 y 0.698 respectivamente, calculados mediante el software SPSS.

\section{Procedimiento}

El diseño metodológico contempló tres fases: la primera medición, la aplicación del estímulo y la segunda medición.

En la primera fase, una vez seleccionadas la población y la muestra se aplicó el cuestionario en la fase de preprueba a fin de indagar el nivel de desarrollo de habilidades cognitivas que presentaban los alumnos y de conocimiento con que contaban en relación a la adición y sustracción de fracciones comunes, así como la correlación existente entre estas dos variables identificadas. 
En la segunda fase, para aplicar el estímulo se recurrió a la aplicación de una secuencia de aprendizaje estructurada en 6 sesiones de trabajo. Cada sesión tuvo una duración de 2 horas y contempló como estrategias principales el juego y el trabajo colaborativo.

Con ayuda de material concreto y manipulable, los alumnos resolvieron operaciones de adición y sustracción de fracciones comunes en el marco de una situación problema real y cotidiana. Dicho material consistió en tablillas de cartulina divididas y recortadas en medios, tercios, cuartos, quintos, sextos, séptimos, octavos, novenos y décimos, elaboradas por ellos mismos.

En la tercera fase se realizó una segunda medición. Para ello se aplicó un segundo cuestionario. Se cuidó que este segundo instrumento no contemplara las mismas fracciones, a fin de que obtener resultados más fiables.

\section{Resultados}

Al realizar una comparación entre los resultados que se obtuvieron en la preprueba y posprueba a partir de estadísticos descriptivos, se identificó una diferencia entre los promedios de los datos que proyectaron las variables y dimensiones, siendo mayor el promedio en la segunda medición, tal como lo indican los siguientes gráficos 1 y 2:

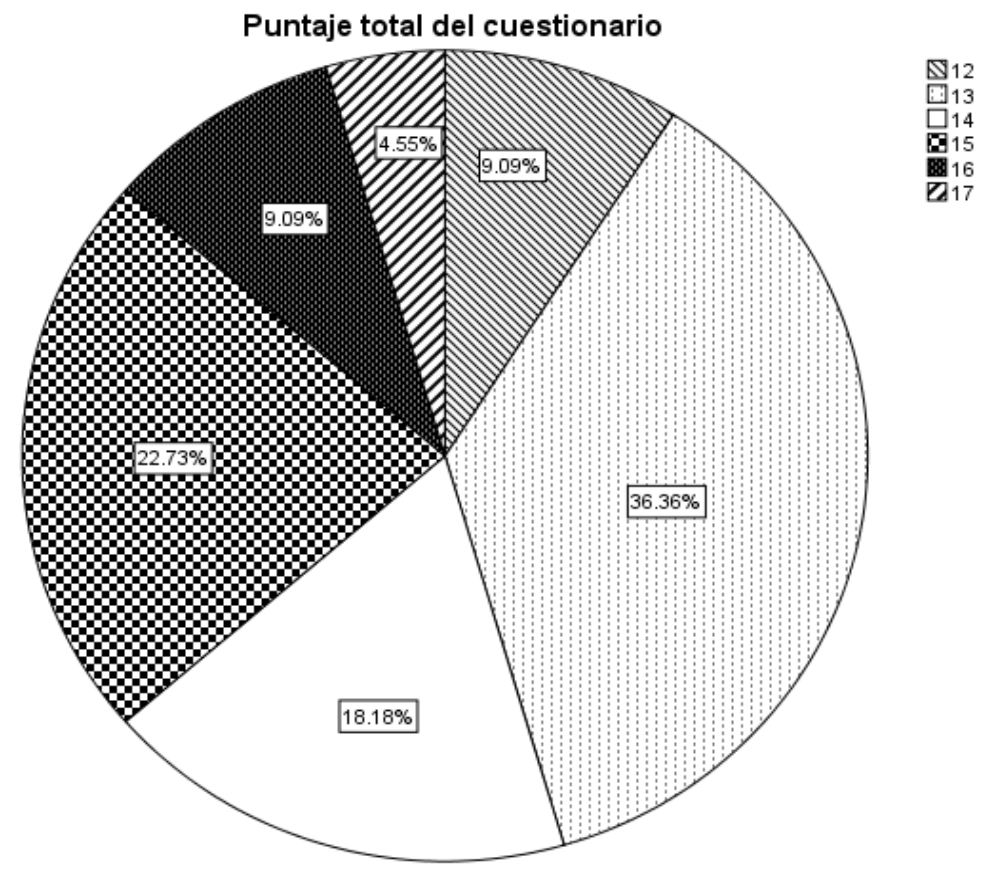

Gráfico 1. Puntajes proyectados por el cuestionario en la fase de la preprueba

El puntaje total del cuestionario fue de 24 puntos. De estos la muestra proyectó un puntaje situado en el intervalo de 12-17 puntos en el que el 9.09\% obtuvo 12 puntos, el 36.36\% 13 puntos, el $18.18 \% 14$ puntos, el $22.73 \% 15$ puntos, el $9.09 \% 16$ puntos y, finalmente, solo el $4.55 \%$ obtuvo máximo puntaje del intervalo referido, es decir, 17 puntos. Con base en lo anterior, el puntaje fluctúa en el $50 \%$ con respecto al puntaje total del cuestionario. Tales resultados denotan el bajo desarrollo de habilidades cognitivas y dificultades para el manejo y solución de la adición y sustracción de fracciones comunes presentes en la muestra. 
Tales datos cuantitativos denotan la poca presencia de saberes previos y de estructura cognitiva e indican la ausencia de significado psicológico con relación al concepto, orden, comparación, equivalencia de fracciones, adición y sustracción de fracciones, todo lo cual se advierte a partir de la dificultad de los sujetos para asignar el significado correcto al signo o símbolo matemático que corresponde a una fracción común determinada y relacionarla con la cantidad o magnitud que aquellas representan. Esto influye en la habilidad para ordenar, comparar y establecer equivalencia de fracciones comunes. Empero, representan conocimientos suficientes que permiten recuperar, relacionar y anclar la nueva información.

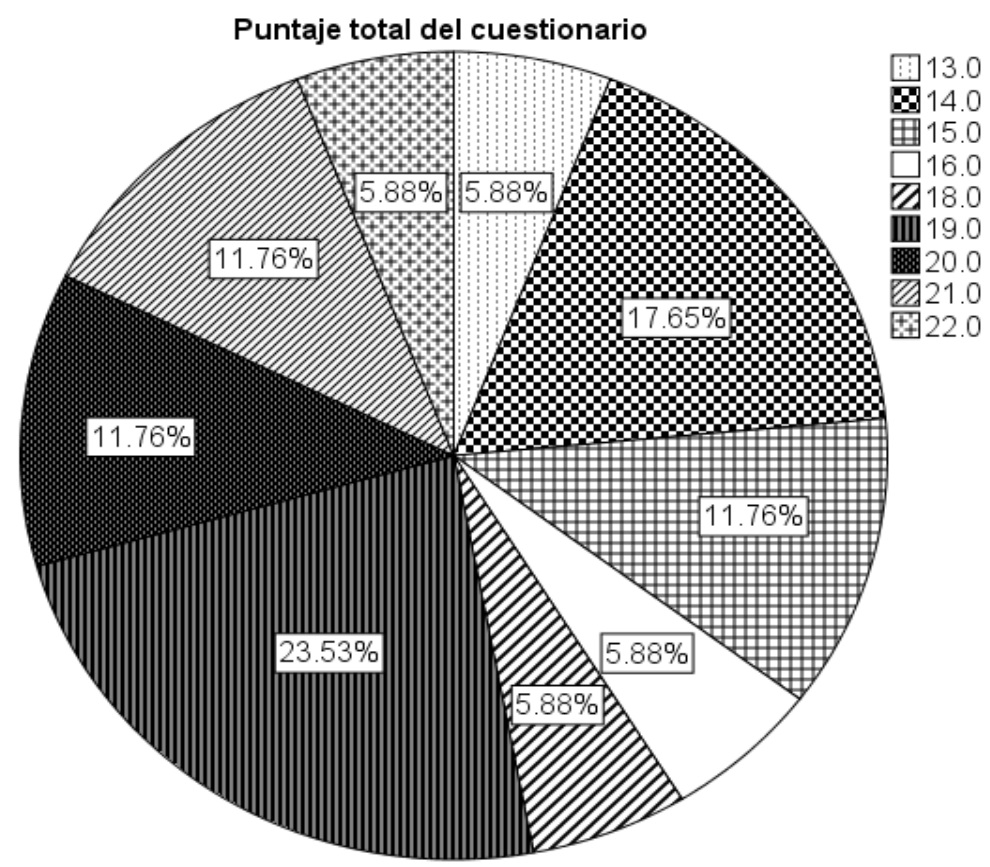

Gráfico 2. Puntajes proyectados por el cuestionario aplicado en la posprueba

Al analizar los datos proyectados por el total de los ítems, dimensiones y variables contemplados en el instrumento de medición, se encontró que los puntajes se inscriben en un rango que fluctúa entre 13 y 22. Con ello es posible señalar que el rango de puntuación del instrumento osciló entre 2 y 24 puntos. Los puntajes más altos con respecto a la preprueba son de 18, 19,20, 21 y 22 con porcentajes de 5.88\%, 23.53\%, 11.76\%, 11.76 y $5.88 \%$ respectivamente. Con base en este rango de puntajes y porcentajes es posible señalar una mejora importante en las dimensiones y variables medidas.

Esta comparación del rango de puntajes y porcentajes entre los datos de la preprueba y posprueba permite señalar una mejora importante en las dimensiones y variables, es decir, reflejan una mejora en el desarrollo de habilidades cognitivas y aprendizaje significativo de la adición y sustracción de fracciones comunes. Esta mejora fue determinada por la estructura cognitiva, los saberes previos que favorecieron el potencial psicológico en los sujetos de la muestra. Con ello es posible determinar que los sujetos lograron asignar significado al signo matemático utilizado para designar una fracción y utilizarlo en la adición y sustracción de fracciones comunes. Asimismo, el objeto de aprehensión fue significativamente lógico en tanto que fue relacionable con la estructura cognitiva del alumno. 
Concerniente a la correlación entre las variables y las dimensiones correspondientes, los datos proyectaron que de las variables desarrollo de habilidades cognitivas y operaciones con fracciones comunes se obtuvo una correlación bilateral de $0.845,0.735$ y 0855 a partir de los estadísticos Pearson, Tau_b de Kendall y Rho de Spearman, respectivamente. Se identificó una correlación positiva, lo cual indica que el comportamiento es igual a la hipótesis formulada: a mayor habilidad cognitiva mayor es el aprendizaje de la adición y sustracción de fracciones. Empero, solo se determinó la interrelación existente entre estas; no se determina la dependencia e independencia entre las mismas. La correlación entre estas variables es más fuerte con los estadísticos $r$ de Pearson y Rho de Spearman dado que con los coeficientes de estos supera los 0.8 . El coeficiente proyectado permite aceptar con una significancia de 0.01 la correlación entre las habilidades cognitivas y las operaciones con fracciones. Por lo anterior se acepta, sin riesgo de error, la hipótesis de investigación y se rechazan la hipótesis nula y la alternativa.

Los coeficientes de correlación obtenidos en relación a las dimensiones contempladas para las variables se muestran en el cuadro $\mathrm{N}^{\circ} 2$.

Tabla 2. Correlación de dimensiones. Coeficientes calculados con un nivel de significancia igual a 0.01 para todos los casos.

\begin{tabular}{|c|c|c|c|}
\hline Correlación de dimensiones & Pearson & $\begin{array}{c}\text { Tau_b de } \\
\text { Kendall }\end{array}$ & $\begin{array}{c}\text { Rho de } \\
\text { Spearman }\end{array}$ \\
\hline $\begin{array}{l}\text { Equivalencia y adición de fracciones } \\
\text { comunes }\end{array}$ & 0.813 & 0.765 & 0.818 \\
\hline $\begin{array}{l}\text { Equivalencia y sustracción de fracciones } \\
\text { comunes }\end{array}$ & 0.692 & 0.696 & 0.775 \\
\hline $\begin{array}{l}\text { Comparación y adición de fracciones } \\
\text { comunes }\end{array}$ & 0.823 & 0.782 & 0.823 \\
\hline $\begin{array}{l}\text { Comparación y sustracción de fracciones } \\
\text { comunes }\end{array}$ & 0.537 & 0.579 & 0.624 \\
\hline Orden y adición de fracciones comunes & 0.468 & 0.510 & 0.561 \\
\hline Orden y sustracción de fracciones comunes & 0.533 & 0.528 & 0.553 \\
\hline
\end{tabular}

La correlación entre las dimensiones equivalencia de fracciones comunes y adición de fracciones comunes es fuerte y positiva. Los coeficientes proyectados permitieron aceptar la correlación entre las dimensiones, en tanto que estadísticamente son diferentes a cero y positivos. Empero, tales coeficientes solo alcanzan el nivel de significación con base en los estadísticos de Pearson y Spearman dado que superan el 0.8. Sin embargo, se acepta la hipótesis de investigación y se rechazan, en consecuencia, las hipótesis nula y alternativa.

La correlación entre equivalencia y sustracción de fracciones comunes es positiva y diferente a cero. Empero, con base en los coeficientes proyectados, el nivel de significancia indica una ligera correlación entre estas, por lo que puede aceptarse con un margen de error. Con respecto a la correlación entre las dimensiones comparación de fracciones comunes y adición de fracciones comunes, la misma es positiva; alcanza un coeficiente diferente a cero. Sin embargo, el nivel de significancia solo se obtiene con los estadísticos Pearson y Spearman. 
Con respecto a comparación de fracciones comunes y sustracción de fracciones comunes los coeficientes admiten una correlación baja y positiva. Evidentemente con ello se comprueba, acepta y fortalece la hipótesis estadística de investigación, dado que la correlación no es igual a cero. Sin embargo, los coeficientes que proyectan los tres estadísticos aplicados -r de Pearson, Rho de Spearman y Tau_b de Kendall- no alcanzan el nivel de significancia, por lo que se reconoce un margen de error al puntualizar la correlación.

La correlación entre las dimensiones ordenar fracciones comunes y adición de fracciones comunes, así como entre las dimensiones ordenar fracciones comunes y sustracción de fracciones comunes, es débil. Aunque la correlación estadísticamente es diferente a cero, esta no alcanzó el nivel de significancia con ninguno de los estadísticos aplicados. El comportamiento de estos datos impide aceptar la hipótesis de investigación sin margen de error.

La correlación positiva y significancia obtenidas entre las variables y dimensiones respectivas permitieron aceptar la hipótesis de trabajo, es decir, enunciar que el aprendizaje significativo de la adición y sustracción de fracciones comunes se ve favorecida por el factor habilidad cognitiva del alumno.

La correlación positiva y significancia obtenidas entre las dimensiones ordenar fracciones comunes, comparar fracciones comunes y equivalencia de fracciones comunes entre las dimensiones adición de fracciones comunes y sustracción de fracciones comunes respectivas señalan que las primeras representan un saber previo a enlazarse con la nueva información. Así, las segundas dimensiones representaron el significado lógico a enlazarse con la estructura cognitiva del sujeto. Tanto saberes previos como la nueva información presentada lograron en el sujeto un significado potencial y psicológico, concretándose de esta manera el aprendizaje significativo de la adición y sustracción de fracciones comunes.

Asimismo, esta correlación indica la asimilación del objeto de aprendizaje dado que tanto los saberes previos como la nueva información evolucionaron, hecho que se advirtió en el desarrollo de la capacidad abstracta para señalar que una fracción no siempre es mayor al significarse con dígitos mayores con respecto a otra y, en sentido contrario, una fracción no siempre es menor al simbolizarse con dígitos menores. A modo de ejemplo determinaron correctamente que 2/6 es menor que 2/4. Asimismo comprendieron que es posible simbolizar un mismo valor con diferentes fracciones lo que les permitió establecer de manera correcta la equivalencia de fracciones, por ejemplo: 4/8 =5/10. Con respecto a la adición y sustracción de fracciones, dejaron de operar bajo la lógica de los números naturales, es decir, sumar numerador con numerador y denominador con denominador.

\section{Comentarios finales}

La correlación entre las variables habilidades cognitivas y operaciones con fracciones comunes es fuerte, positiva y con una significancia de 0.01. Esto indica que los casos analizados lograron desarrollar las habilidades cognitivas correspondientes al orden, comparación y equivalencia de fracciones comunes y denota el impacto positivo de las mismas en la solución de la adición y sustracción de fracciones comunes. En otras palabras, favorecen la apropiación de saberes nuevos, la mejora de los mismos, la asignación de sentido y significatividad a conceptos, nociones, ideas o conocimientos social y culturalmente compartidos. Asimismo son útiles para el desarrollo cognitivo del sujeto. De esta manera se supera el aprendizaje repetitivo y memorístico, sin razonamiento y sin análisis del algoritmo de tales números o dígitos y la resolución de operaciones aludidas. 
El desarrollo de habilidades cognitivas tales como comparar y ordenar es fundamental dado que las mismas permiten al sujeto la apropiación clara del sentido de magnitud o cantidad exacta que representa una fracción. Esto permite, entonces, dotar del significado correcto que la cultura y el conocimiento matemático han asignado a la fracción, a la conformación de una fracción por dos dígitos, de la misma manera en la que opera mentalmente con los números naturales cuando se percata de que al 2, como símbolo, se le asigna el significado de representar una cantidad mayor a 1 y menor a 3. Supera con ello la noción errónea de asignar siempre un valor, cantidad o magnitud mayor a una fracción formada por dígitos mayores con respecto a otra conformada por números menores. A modo de ejemplo: establecer que $2 / 6$ es mayor que $1 / 2$ cuando realmente $1 / 2$ representa una cantidad, valor o magnitud mayor con respecto a 2/6. Ello denota en el sujeto la adquisición de sentido numérico.

Una vez que el sujeto desarrolló tales nociones y las comprendió, mostró capacidad para comparar y ordenar fracciones comunes así como para establecer equivalencia entre estas en términos abstractos, comprendió el algoritmo tradicional para la solución de la adición y sustracción de fracciones comunes, es decir que para realizar estas tareas prescindió del uso de recursos concretos.

Los recursos didácticos concretos, palpables y manejables utilizados en el proceso de enseñanza favorecieron el criterio de relacionalidad y el significado lógico del objeto de aprehensión. Estos favorecieron la relación sustancial entre la nueva información y la estructura cognitiva del alumno, lo cual tuvo un impacto favorable en la adquisición de significados psicológicos. Esto resulta comprobable por la mayor facilidad de manejo del algoritmo de la adición y sustracción de fracciones que mostró el sujeto. Asimismo, esto coadyuvó a comprender que estas operaciones matemáticas no se realizan bajo la lógica de los números naturales.

Finalmente, las características de los recursos didácticos aludidos favorecieron la disposición del sujeto para que el objeto de aprendizaje fuese potencialmente significativo. Con ello, la variable motivación representó uno de los elementos determinantes para que la muestra analizada lograse la comprensión de fracciones y la lógica de las operaciones matemáticas involucradas, es decir, para que se lograse un aprendizaje significativo de dichas operaciones que en su momento no se lograron comprender.

\section{Referencias bibliográficas}

Ausubel, D. P.; Novak, J. D. \& Hanesian, H. (2009). Psicología educativa, un punto de vista cognoscitivo. México, D.F.: Trillas.

Ávila Storer, A. (2006). Prácticas cotidianas y conocimiento sobre las fracciones. Estudio con adultos de escasa o nula escolaridad. Educación Matemática, 18(1), 5-33. Recuperado de http://www.revista-educacion-matematica.com/descargas/Vol18-1.pdf

Baena, G. (1986). Instrumentos de investigación. México: Editores Mexicanos Unidos.

Block, D. \& Solares, D. (2001). Las fracciones y la división en la escuela primaria: análisis didáctico de un vínculo. Educación Matemática, 13(2), 5-30. México: Grupo Editorial Iberoamérica. Recuperado de http://www.revista-educacion-matematica.org.mx/ descargas/Vol13/2/02Block.pdf 
Blumen, S. (1997). El desarrollo de las habilidades cognitivas según los avances en las teorías psicológicas. Revista de psicología de la PUCP, 15(1). Recuperado de http://revistas. pucp.edu.pe/index.php/psicologia/article/view/4572/4551

Coll Salvador, C. (1990). Aprendizaje escolar y construcción del conocimiento. Barcelona: Paidos Ibérica.

Hernández Sampieri, R.; Fernández-Collado, C. \& Baptista Lucio, P. (2010). Metodología de la investigación. México: Mc. Graw Hill.

Kerlinger, F. N. \& Lee, H. B. (2002). Investigación del comportamiento. Técnicas y metodología. México: Mc. Graw Hill/Interamericana Editores.

López García, J. C. (2014). La taxonomía de Bloom y sus actualizaciones. Eduteka. Recuperado de http://eduteka.icesi.edu.co/articulos/TaxonomiaBloomCuadro

Mora De la, L. (1977). Psicología del aprendizaje. Formas 2. México: Progreso.

Mora De la, L. (1979). Psicología del aprendizaje. Teorías 1. México: Progreso.

Parra Álvarez, M. Á. \& Flores Macías, R. C. (2008). Aprendizaje cooperativo en la solución de problemas con fracciones. Educación Matemática, 20(1), 31-52. México: Grupo Santillana. Recuperado de http://www.redalyc.org/articulo.oa?id=40512063003

Perera Dzul, P. B. \& Valdemoros Álvarez, M. E. (2009). Enseñanza experimental de las fracciones en cuarto grado. Educación matemática, 21(1), 29-61. México: Grupo Santillana. Recuperado de http://www.scielo.org.mx/scielo.php?script=sci_arttext\&pid=S1665$\underline{58262009000100003 \& \operatorname{lng}=e s \& t \operatorname{lng}=e s}$

Pozo, J. I. (2006). Teorías cognitivas del aprendizaje. Madrid: Morata.

Rivas, M. (2010). Procesos cognitivos y aprendizaje significativo. Madrid: Organización Educativa de la Comunidad de Madrid.

Schunk, D. H. (2012). Teorías del aprendizaje. Una perspectiva educativa. Traducción Leticia E. Pineda Ayala y María Elena Ortiz Salinas. México: Pearson educación.

Terán de Serrantino, M. \& Pachano Rivera, L. (2009). El trabajo cooperativo en la búsqueda de aprendizajes significativos en clase de matemáticas de la educación básica. Educere, 13(44), 159 - 167. Recuperado de http://www.saber.ula.ve/bitstream/123456789/28852/3/ investiga4.pdf 